\title{
On model of microstructure formation during selective laser melting of metal powder bed
}

\author{
F.Kh. Mirzade ${ }^{1}$, A.V. Dubrov ${ }^{1}$ \\ ${ }^{I}$ Institute on Laser and Information Technologies - Branch of Federal Scientific Research Centre "Crystallography and Photonics" of Russian Academy of \\ Sciences, 140170, Shatura, Russia
}

\begin{abstract}
A model of phase field has been developed to investigate the microstructure evolution during selective laser melting (SLM) of metal powder bed. A two-component (degree of order, orientation field) ordering parameter has been used, for which the permissive relationships have been derived reasoning from the principle of entropy production positivity. The application of this principle has made possible obtaining the thermodynamically agreed evolutionary equations for the components of the ordering parameter, conjugate with the fields of temperature, admixture concentration and elastic deformations for the non-isothermal conditions of crystallization of pure metal melts and multicomponent alloys. The model of the microstructure is adjoint with the macroscopic thermodynamic model of SLM that accounts for the processes of heat transfer, thermo-capillary convection and evolution of the melt free surface.
\end{abstract}

Keywords: additive manufacturing; selectiv laser melting; powder bed; phase field method; multiscale model; microstructure; elastic stresses

\section{Introduction}

Powder-bed selective laser melting (SLM) is a promising metal additive manufacturing (AM) technique for producing complex structures out of powders (or their mixtures) in a layer by layer fashion The process creates 3D solid objects by bonding powdered materials using laser beam energy [1-4]. This technology is practically non-waste and universal, as it makes use of a wide enough range of initial powders with the sizes of particles from $10 \mathrm{~nm}$ to $100 \mu \mathrm{m}[1,2]$. The SLM process is determined by a large number of factors, such as energy source power, scanning speed, physical and chemical properties of the initial material, etc. Noteworthy also are the interrelation of factors affecting the process and the presence of many interacting processes: absorption and scattering of laser radiation energy by the substrate matter and powder particles, heat conduction and convection, evolution of the melt free surface at the cost of capillary (thermo-capillary as well) forces, evaporation, shrinkage, crystallization, formation of the microstructure and stressed state of the synthesized object [4].

It is known from the experiments that various microstructures (cellular, dendritic, cellular-dendritic structures) emerging at the stage of melt crystallization define to a large extent the physical and mechanical characteristics of the product to be synthesized, so it is essential that the process of microstructure formation should be controlled. The best applicable basis to exert this control is mathematical simulation that allows for establishing a linkage between the SLM process parameters and the quality of the build parts.

Unified simulation of crystallization in SLM involves serious difficulties. The main problems are related to the description of the complex interaction of the nonlinear processes taking place at different scale levels, from the level of interaction between a single growing crystallite and the metastable melt to the macro-level (the description of heat-mass transfer at the level of the whole system). The existing models of crystallization process developed in the framework of one scale level are capable of describing rather complex phenomena (formation of dendrites, growth of crystalline grains, porosity, etc.) at their levels. Despite this, nevertheless, the recent new lines of investigations deal with joint models of micro- and macro-levels [5].

Our previous work [6] has suggested a multiscale model of the processes of crystallization and microstructure evolution in laser surfacing with coaxial injection of metal and alloy powders. In [7], consideration is given to the macromodel of the processes that is a part of the multilevel model of crystallization in laser surfacing. Work [8] presents a numerical multiscale model of melting of the metal powder layer for the conditions of permanent heat flows.

The present work is aimed at the development of a model of solid-phase microstructure formation in SLM of a powder bed, applying two-scale approximation. The essence of the model is that in the physico-mathematical description of the crystallization problem the physical processes are presented as a group of related processes progressing at different spatial scales and exhibiting mutual influence. At each level, a model of crystallization process is developed that allows for the features of melt behavior at this level. This fact defines the range of problems for submodels as well.

The microstructure evolution is described by the equation for the two-component (degree of order, orientation field) ordering parameter, conjugate with the equations of heat conduction and admixture diffusion, as well as with elastic stress/deformation that accompanies the phase transformation (PT). The microstructure model is adjoint with the macroscopic thermodynamic model of SLM that accounts for the processes of heat transfer, thermo-capillary convection and evolution of the melt free surface (liquid-gas interfaces). The macromodel gives self-consistent consideration to the distribution of temperature and melt velocities depending on the SLM process parameters (beam power, scanning speed, powder layer). Modeling of the free surface evolution is performed by the method of volume of fluid (VOF). 


\section{Phase field model}

The investigation of microstructures in melt crystallization using the classical (Stefan-type) model presents a rather difficult task, as it calls for the development of special algorithms for an explicit definition of the shape of the interface. Best suited for this purpose is the employment of the continuum model of phase field (MPF) relying on Landau-Ginzburg principles of weakly non-equilibrium thermodynamics and formalism of PT.

In contrast to the classical model that uses the notion of a sharp boundary, the continuum model follows the concept of a diffusion interface between the liquid and solid phases. With this approach, the shape and relative position of the phases making up the microstructure are described by the variables of the phase field (or of the ordering parameter), which are governed by a set of nonlinear differential equations conjugate with the equations of heat conduction and concentration. The ordering parameter is smoothly varied over the width of the narrow transition area, describing the inner structure of PT. Away from the interface it has a constant value corresponding to the structure, orientation and their composition. Therefore, the MPF is a convenient instrument for the numerical investigation of crystallization that does not require explicit tracking of the interface in the course of the microstructure evolution; the phase boundary position is here determined as the phase field isoline.

The MPF was applied to a wide range of problems, including the growth of dendrites in pure metals; dendritic, eutectic and peritectic growth in alloys; microsegregation of the admixture on fast solidification, etc. The thermodynamically agreed MPF were considered in a number of papers [9-13]. The derivation of evolutionary equations in these works is based on the main principles of irreversible thermodynamics. To describe the melt crystallization (micro-level problems) in SLM, we represent the derivation of the dynamic equations for the MPF with regard to elastic (thermal, concentration, and phase) stresses accompanying the process of non-isothermal PT.

To obtain the governing equation of the MPF, make use of the formalism suggested in [9]. Consider an arbitrary region having volume $V$, where the binary metal $(a-b)$ undergoes the liquid-solid (L-S) PT. Restrict ourselves to the 2D variant of the problem and introduce the characteristic of the material phase state - the ordering parameter consisting of two variables $\{\varphi(\mathbf{r}, t), \phi(\mathbf{r}, t)\}[14]$. The variable $\varphi(\mathbf{r}, t)$ can be interpreted as the degree of the material ordering in the microvolume with the radius-vector $\mathbf{r}$ at the moment of time $t ; \phi=0$ corresponds to the liquid state, and $\phi=1-$ to the crystalline state. The narrow region, where $0<\varphi<1$, corresponds to the phase interface. The $\phi$ variable describes the crystalline phase orientation (crystallization orientation field). It is determined as $\phi=N_{0} \phi$, where $\phi$ is angle between one of the main crystallographic directions and the $\mathrm{X}$-axis in the chosen coordinate system, $N_{0}$ is the order of the symmetry axis of the grating type under study. It is apparent that $\phi \in\left(0,2 \pi / N_{0}\right)$.

For an arbitrary subvolume of the region $\Omega \in V$ under consideration the functional of total entropy will be written as

$$
E(\Omega)=\int_{\Omega}\left[\eta(\varphi, c, u)-\frac{1}{2} \varepsilon^{2}|\nabla \varphi|^{2}-\frac{1}{2} v^{2}|\nabla \phi|^{2}\right] d v
$$

where $\eta(\varphi, c, u)$ is the entropy density; $u(\mathbf{r}, t)$ is the internal energy density; $c$ is the concentration of the dissolved matter (admixture): $\varepsilon$ and $v$ are the positive parameters, which can be the functions of the ordering parameter. The gradient terms in (1) allow for the contributions to entropy at the cost of the interphase boundaries. In the employed functional, the gradients of ordering parameters are only taken into account; the gradients of temperature and concentration in the explicit form are not included (they are supposed to be smallish).

The anisotropy of the surface energy can be taken into consideration, supposing that the coefficient $\varepsilon$ is a function of $\theta$, $\varepsilon=\varepsilon_{0} \varepsilon(\theta)$ where $\varepsilon_{0}>0, \theta=\theta_{0}-\phi / N_{0}$. The variable $\theta_{0}$ is the angle between the X-axis and the normal vector $\mathbf{n}=\nabla \varphi$ at the interface. Thus, $\theta$ characterizes the orientation of the interface normal vector in relation to the neighboring growing crystallite. The third term in (1) takes account of the influence of misorientation of the neighboring crystals. It is supposed that this influence depends on the material ordering, so for $v$ we have $v=v_{0} v(\varphi), v_{0}>0$.

Applying the local conservation laws for concentration and energy, as well as the second law of thermodynamics, obtain

$$
\begin{aligned}
& u+\nabla \cdot \mathbf{q}-\sigma_{i j} e_{i j}=0, \\
& c+\nabla \cdot \mathbf{j}=0,
\end{aligned}
$$

where $\mathbf{j}=M_{c} \nabla(\delta E / \delta c)$ and $\mathbf{q}=M_{u} \nabla(\delta E / \delta u)$ are the fluxes of concentration and energy, respectively; $M_{c, u}>0$ are the constants describing the dissolved substance diffusion and heat conduction; $\sigma_{i j}$ and $e_{i j}$ are the tensors of stresses and deformations, respectively. The third term in (2) characterizes the variation of inner energy due to elastic deformation.

Entropy production in the volume $\Omega \in V$ can be calculated by subtracting the entropy flow through the surface from the rate of entropy variation $E$ in $\Omega \in V$ :

$$
G_{\text {prod }}=E+\int_{A}\left(\frac{\mathbf{q}}{T}+\mathbf{p}\right) \cdot \mathbf{n} d a,
$$

where $A$ is the surface of $\Omega$ with the outer normal $\mathbf{n}$,

$$
\mathbf{p}=\varphi\left[\varepsilon^{2} \nabla \varphi+\varepsilon \varepsilon^{\prime} I \cdot \nabla \varphi\right]+\phi v \nabla \phi
$$


( $I$ is the unit tensor, $\varepsilon^{\prime}=d \varepsilon(\theta) / d \theta$ ). In the integrand in (4), $\mathbf{q} / T$ is the entropy flow due to heat conduction, $\mathbf{p}$ is the entropy flux related to changing of phase variables (degree of order, orientation of the growing crystal) at the boundary of $\Omega$ volume.

By substituting expression (1) into equation (4) and applying the divergence theorem, find for the second law of thermodynamics $\left(G_{\text {prod }} \geq 0\right)$

$$
\int_{\Omega}\left[\eta+\nabla \cdot\left(\frac{\mathbf{q}}{T}\right)+h_{\varphi} \varphi+h_{\phi} \phi\right] d v \geq 0,
$$

where the following designations are introduced:

$$
\begin{aligned}
& h_{\varphi}=\nabla \cdot\left[\varepsilon\left(\varepsilon I+\varepsilon^{\prime} J\right) \nabla \varphi\right]-v v^{\prime}|\nabla \phi|^{2}, \\
& h_{\phi}=\varepsilon \varepsilon^{\prime}|\nabla \varphi|^{2}+\nabla \cdot(v \nabla \phi)
\end{aligned}
$$

$\left(J=\mathbf{i}+\mathbf{j} \mathbf{j}\right.$ is the tensor with the orthonormal basis $(\mathbf{i}, \mathbf{j})$ in the Cartesian coordinate system, $\left.v^{\prime}=d v(\varphi) / d \varphi\right)$. Hence, we have the local expression

$$
\eta+\nabla \cdot\left(\frac{\mathbf{q}}{T}\right)+h_{\varphi} \varphi+h_{\phi} \phi \geq 0
$$

Further, applying the law of conservation of energy (2) and using Gibbs equation for free energy $g=u-T \eta-\sigma_{i j} e_{i j}$, write inequality (6) in the form:

$$
-g-\eta T-\left(\frac{\mathbf{q}}{T} \cdot \nabla T\right)+h_{\varphi} \varphi+h_{\phi} \phi-e_{i j} \sigma_{i j} \geq 0 \text {.(7) }
$$

The time derivative of free energy $(g)$ is represented as

$$
g=\frac{\partial g}{\partial T} T+\frac{\partial g}{\partial \sigma_{i j}} \sigma_{i j}+\frac{\partial g}{\partial \varphi} \varphi
$$

Then inequality (7) takes the form:

$$
\left(h_{\varphi} T-\frac{\partial g}{\partial \varphi}\right) \varphi+h_{\phi} \phi T-\left(\eta+\frac{\partial g}{\partial T}\right) T-\left(\frac{\mathbf{q}}{T} \cdot \nabla T\right)+\left(e_{i j}+\frac{\partial g}{\partial \sigma_{i j}}\right) \sigma_{i j} \geq 0 .
$$

The positivity of entropy production can be locally assured having chosen the following relationships for the thermal flow and the time derivatives of variables of the ordering parameter:

$$
\begin{aligned}
& \mathbf{q}=M_{e} \nabla \frac{1}{T}, \\
& \tau \varphi=\nabla \cdot\left[\varepsilon\left(\varepsilon I+\varepsilon^{\prime} J\right) \nabla \varphi\right]-\frac{v^{\prime}}{2}|\nabla \phi|^{2}-\frac{1}{T} \frac{\partial g}{\partial \varphi}, \\
& \tau \phi=\varepsilon \varepsilon^{\prime}|\nabla \varphi|^{2}+\nabla \cdot(v \nabla \phi),
\end{aligned}
$$

where $\eta=-\partial g / \partial T, e_{i j}=-\partial g / \partial \sigma_{i j}, \tau=\tau_{0} \tau(\theta)$ is the function describing mobility.

Equations (2), (3) and (8)-(10) represent the set of master equations for the phase field, admixture concentration and energy.

For the densities of Gibbs free energy $(g(\varphi, c, T))$ and internal energy $(u(\varphi, c, T))$ in case of regular binary alloys we have the expressions [10]

$$
\begin{aligned}
& g(\varphi, c, T)=(1-c) g_{a}\left(\varphi, T, e_{i j}\right)+c g_{b}\left(\varphi, T, e_{i j}\right)+\lambda(\varphi) c(1-c)+\frac{R T}{v_{m}}[c \ln c+(1-c) \ln (1-c)], \\
& u(\varphi, c, T)=(1-c) u_{a}(\varphi, T)+c u_{b}(\varphi, T),
\end{aligned}
$$

where $g_{a, b}$ and $u_{a, b}$ are the classical densities of free energy and inner energy of the substances $a$ and $b$, respectively; $R$ is the gas constant; $v_{m}$ is the molar volume; $\lambda(\varphi)$ is the alloy imperfection parameter. The densities of inner energies of the substance $a$ and $b$ are written as

$$
u_{a, b}(\varphi, T)=p(\varphi) u_{a, b}^{S}(T)+u_{a, b}^{L}(T)(1-p(\varphi))
$$

where $u_{a, b}^{S, L}(T)$ are the internal energies of the solid and liquid phases of the substances $a$ and $b$ at the temperature $T$; the interpolation function $p(\varphi)$ determines the dependence of the internal energy on the medium order. It is chosen in such a way as to offer a description of the interface $L-S$ of a finite width, where $0<\varphi<1$ (the free energy potential has its minima at $\varphi=0$ and $\varphi=1)$. In accordance with [9]: $p(\varphi)=\varphi^{3}\left(10-15 \varphi+6 \varphi^{2}\right), p(0)=0$ and $p(1)=1$.

Further, representing $u_{a, b}^{S, L}(T)$ as the linear dependences on the temperature: $u_{a, b}^{S, L}(T)=u_{a, b}^{S, L}\left(T_{m}^{a, b}\right)+C_{a, b}^{S, L}\left(T-T_{m}^{a, b}\right)$, where $u_{a, b}^{S}\left(T_{m}^{a, b}\right)$ are the inner energies of the solid and liquid phases at the melting temperature $T=T_{m}^{a, b}, C_{a, b}^{S, L}$ are their heat capacities, we have

$$
u_{a, b}(\varphi, T)=u_{a, b}^{S}\left(T_{m}^{a, b}\right)+C_{a, b}\left(T-T_{m}^{a, b}\right)+p(\varphi) L_{a, b}
$$


( $L_{a, b}=u_{a, b}^{S}\left(T_{m}^{a, b}\right)-u_{a, b}^{L}\left(T_{m}^{a, b}\right)=T_{m}^{a, b} \eta_{a, b}^{S}-\eta_{a, b}^{L}$ is the latent heat of the components, $\left.C_{a, b}^{S}=C_{a, b}^{L}=C_{a, b}\right)$. Thereafter, using the thermodynamic relation $d g=-s d T+\sigma_{i j} d e_{i j}$ for the densities of free energies of the components $g_{a, b}$ after integrating obtain

$$
g_{a, b}=\omega_{g}^{a, b} T d(\varphi)+\left[L_{a, b}\left(1-T / T_{m}^{a, b}\right)+\vartheta_{a, b}\left(e_{i j}\right)\right] p(\varphi) .
$$

Here, $\omega_{g}^{a, b}=3 \bar{\sigma}_{a, b} / \sqrt{2} T_{m}^{a, b} \delta_{a, b}\left(\bar{\sigma}_{a, b}\right.$ is the surface energy of the $L-S$ boundary; $\delta_{a, b}$ is the PT front width (the typical scale of the phase field length) is the height of the energy barrier related to the interphase boundary $(L-S) ; d(\varphi)$ is the double-wall potential. The term $\vartheta_{a, b}\left(e_{i j}\right)$ in the right side of (13) describes the effect of the elastic fields of deformations on the potential, which is due to PT:

$$
\vartheta_{a, b}\left(e_{i j}\right)=\int_{0}^{e_{i j}}\left(\sigma_{i j}^{S}-\sigma_{i j}^{L}\right) d e_{i j} .
$$

Then, making use of the thermodynamic relation $(\partial \eta / \partial \varphi)_{u, c}=-T^{-1}(\partial g / \partial \varphi)_{T, c}$ find that the gradient of entropy density is

$$
\partial \eta / \partial \varphi=-(1-c) \Gamma_{a}-c \Gamma_{b}-T^{-1} \lambda^{\prime} c(1-c),
$$

where $\Gamma_{a, b}=\omega_{g}^{a, b} d^{\prime}(\varphi)+30 d(\varphi) T^{-1}\left[L_{0}^{a, b}\left(1-T / T_{m}^{a, b}\right)+\vartheta_{a, b}\left(e_{i j}\right)\right], \quad \lambda^{\prime}=d \lambda(\varphi) / d \varphi=30\left(\lambda_{L}-\lambda_{S}\right) d(\varphi)$. In deriving (14), we have taken into account that $\lambda(\varphi)=\lambda_{S}+\left(\lambda_{L}-\lambda_{S}\right) p(\varphi)$ and $p^{\prime}(\varphi)=30 d(\varphi)$.

We have the following expressions for the energy flows $(\mathbf{q})$ and impurity concentration $(\mathbf{j})$, respectively:

$$
\begin{aligned}
& \mathbf{q}=-\frac{M_{e}}{T^{2}} \nabla T, \\
& \mathbf{j}=D(\varphi) \frac{c(1-c) v_{m}}{R}\left[\left(\Gamma_{a}-\Gamma_{b}-T^{-1} \lambda^{\prime}(1-2 c)\right) \nabla \varphi-p(\varphi)\left(\vartheta_{a}^{\prime}-\vartheta_{b}^{\prime}\right) \nabla e_{i j}\right] \\
& \quad-D(\varphi)\left[1-\frac{2 c(1-c) v_{m}}{R T} \lambda(\varphi)\right] \nabla c,
\end{aligned}
$$

where $\vartheta_{a, b}^{\prime}=d \vartheta_{a, b} / d e_{i j} ; D(\varphi)=D_{S}+p(\varphi)\left(D_{L}-D_{S}\right)$ is the diffusion coefficient that is linked to the $M_{c}$ parameter by the relationship: $M_{c}=D(\varphi) v_{m} c(1-c) R^{-1}$.

Substituting (11), (12) and (13)-(16) into (2), (3) and (9), obtain the final forms of the governing equations for the phase field

$$
\begin{aligned}
\tau \varphi=\varepsilon_{0}^{2} \nabla & \cdot\left[\varepsilon(\theta)\left(\varepsilon(\theta) I+\varepsilon^{\prime}(\theta) J\right) \cdot \nabla \varphi\right]-v_{0} v(\varphi) v^{\prime}(\varphi)|\nabla \phi|^{2} \\
& -(1-c) \Gamma_{a}\left(\varphi, T, e_{i j}\right)-c \Gamma_{b}\left(\varphi, T, e_{i j}\right)-T^{-1} \lambda^{\prime} c(1-c),
\end{aligned}
$$

orientation field

$$
\tau \phi=\varepsilon_{0}^{2} \varepsilon(\theta) \varepsilon^{\prime}(\theta)|\nabla \varphi|^{2}+v_{0} \nabla \cdot(v(\varphi) \nabla \phi),
$$

energy

$$
C T=\chi \nabla^{2} T-\left[p^{\prime}(\varphi) L+\omega_{e} d^{\prime}(\varphi)\right]+3 \kappa T \alpha_{T} \delta_{i j} e_{i j},
$$

and concentration

$$
\begin{aligned}
c= & \nabla \cdot D\left[1-\frac{2 c\left(1-c v_{m}\right.}{R T} \lambda(\varphi)\right] \nabla c+ \\
& +\nabla \cdot\left[M_{c}\left(\Gamma_{b}-\Gamma_{a}+T^{-1} \lambda^{\prime}(1-2 c)\right) \nabla \varphi+p(\varphi)\left(\vartheta_{a}^{\prime}-\vartheta_{b}^{\prime}\right) \nabla e_{i j}\right],
\end{aligned}
$$

where $\chi=M_{e} T^{-2}=(1-c) \chi_{a}+c \chi_{b}$ is the heat conduction, $C=(1-c) C_{a}+c C_{b}, L=(1-c) L_{a}+c L_{b}$.

If the thicknesses of the interphase boundaries of the binary alloy components are $\delta_{a}=\delta_{b}=\delta$, the phase field equation (17) is much simplified and takes the form:

where

$$
\begin{aligned}
\tau \varphi=\varepsilon_{0}^{2} \nabla \cdot & {\left[\varepsilon(\theta)\left(\varepsilon(\theta) I+\varepsilon^{\prime}(\theta) J\right) \cdot \nabla \varphi\right]-v_{0}^{2} v(\varphi) v^{\prime}(\varphi)|\nabla \phi|^{2} } \\
& -\varepsilon_{0}^{2}\left[\frac{1}{\delta^{2}} \xi_{1}(c) p^{\prime}(\varphi)+\xi_{2}\left(c, T, e_{i j}\right) d^{\prime}(\varphi)\right]-T^{-1} \lambda^{\prime} c(1-c),
\end{aligned}
$$

$$
\begin{aligned}
& \varepsilon_{0}^{2}=6 \sqrt{2} \delta\left(\bar{\sigma}_{a}+\bar{\sigma}_{b}\right)\left(T_{m}^{a}+T_{m}^{b}\right)^{-1}, \xi_{1}(c)=(1-c) \xi_{1}^{a}+c \xi_{1}^{b}, \xi_{2}(c, T)=(1-c) \xi_{2}^{a}(T)+c \xi_{2}^{b}(T), \\
& \xi_{1}^{a, b}=\frac{2 \bar{\sigma}_{a, b}}{\bar{\sigma}_{a}+\bar{\sigma}_{b}}\left(1+T_{m}^{b, a} / T_{m}^{a, b}\right), \quad \xi_{2}^{a, b}\left(T, e_{i j}\right)=\frac{1}{\varepsilon_{0}^{2} T}\left[L_{a, b}\left(1-T / T_{m}^{a, b}\right)+\vartheta_{a, b}\left(e_{i j}\right)\right] .
\end{aligned}
$$

In this case, equation (20) for the concentration is reduced as follows

$$
\begin{aligned}
c=\nabla \cdot D & {\left[1-\frac{2 c(1-c) v_{m}}{R T} \lambda(\varphi)\right] \nabla c+\nabla \cdot\left[m _ { 0 } \left(\frac{1}{\delta} \xi_{1}^{\prime}(c) p^{\prime}(\varphi)+30 \delta \xi_{2}^{\prime}\left(c, T, e_{i j}\right) p(\varphi)\right.\right.} \\
& \left.\left.+T^{-1} \lambda^{\prime}(1-2 c)\right) \nabla \varphi+p(\varphi)\left(\vartheta_{a}^{\prime}-\vartheta_{b}^{\prime}\right) \nabla e_{i j}\right],
\end{aligned}
$$

where 


$$
m_{0}=\frac{6 \sqrt{2} v_{m}\left(\bar{\sigma}_{a}+\bar{\sigma}_{b}\right)}{R\left(T_{m}^{a}+T_{m}^{b}\right)}, \quad \xi_{1}^{\prime}(c)=d \xi_{1}(c) / d c, \quad \xi_{2}^{\prime}(c)=d \xi_{2}(c) / d c .
$$

The fields of elastic strains can be expressed in terms of the phase field relying on the condition of mechanical equilibrium:

$$
\nabla_{j} \sigma_{i j}=0, \quad \sigma_{i j}=\sigma_{i j}^{S} p(\varphi)+\sigma_{i j}^{L}(1-p(\varphi)) .
$$

The stress tensors of mono phases are represented as

$$
\sigma_{i j}^{S, L}=\lambda^{S, L} \delta_{i j} \nabla \cdot \mathbf{u}+2 \mu^{S, L} e_{i j}-3 \kappa \delta_{i j}\left[\alpha_{c}^{S, L}\left(c-c_{0}\right)+\alpha_{T}^{S, L}\left(T-T_{0}\right)+\gamma_{v}\right],
$$

where $\lambda^{S, L}$ and $\mu^{S, L}$ are Lamé moduli of elasticity, $\kappa^{S, L}=\lambda^{S, L}+2 \mu^{S, L} / 3$ is the isothermal compression modulus, $\alpha_{c}^{S, L}$ and $\alpha_{T}^{S, L}$ are the coefficients of volumetric concentration and thermal expansions, respectively. In (22), the last term including $\gamma_{v o l}$ allows for the stresses generated because of the difference in the volumes of $L$ and $S$ phases. $\nabla \cdot \mathbf{u}=e_{i i}=\partial u_{i} / \partial x_{i}(\mathbf{u}$ is the vector of elastic displacement).

$$
\begin{aligned}
\sigma_{i j}= & \kappa(\varphi)(\nabla \cdot \mathbf{u}) \delta_{i j}+2 \mu(\varphi)\left(u_{i j}-\frac{1}{d} \delta_{i j} \nabla \cdot \mathbf{u}\right)-3 \kappa^{l} \delta_{i j}\left[\alpha_{c}(\varphi)\left(c-c_{0}\right)\right. \\
& \left.+\alpha_{T}(\varphi)\left(T-T_{0}\right)+\gamma_{v}(\varphi) \kappa_{0}\right],
\end{aligned}
$$

where

$$
\begin{aligned}
& \kappa(\varphi)=\kappa^{l}+p(\varphi) \Delta \kappa, \mu(\varphi)=\mu^{L}+p(\varphi) \Delta \mu, \alpha_{c, T}(\varphi)=\alpha_{c, T}^{L}+p(\varphi) \Delta \alpha_{c, T}, \gamma_{v}(\varphi)=\gamma_{0} p(\varphi), \\
& \Delta \kappa=\kappa^{S}-\kappa^{L}, \quad \Delta \mu=\mu^{S}-\mu^{L}, \quad \Delta \alpha_{c, T}=\kappa_{0} \alpha_{c, T}^{S}-\alpha_{c, T}^{L}, \quad \kappa_{0}=\kappa^{S} / \kappa^{L} .
\end{aligned}
$$

Under certain assumptions, several existing models of the phase field can be produced from the obtained micromodel. For instance, the removal of the equations for orientation field, diffusion and stresses from the model (17)-(21), reasoning that $\phi=0, c=0$ and $\sigma_{i j}=0$, will result in producing the model of pure substance crystallization (Wang and coauthors [9]). If $T=$ const $, \phi=0, \sigma_{i j}=0$, as well as $\lambda(\varphi)=0$ (an ideal alloy), the Warren and Boettinger [10] model for isothermal crystallization of binary alloys will be obtained.

In the case that the elastic properties of the liquid and solid phases on PT are identical $(\Delta \kappa=\Delta \mu=0)$, and the variations of the temperature and admixture concentration are insignificant $\left(T=T_{0}, c=c_{0}\right)$, the phase field model is considerably simplified, and for 2D systems, it takes the form:

$$
\begin{gathered}
\tau \varphi=\varepsilon_{0}^{2} \nabla \cdot\left[\varepsilon(\theta)\left(\varepsilon(\theta) I+\varepsilon^{\prime}(\theta) J\right) \cdot \nabla \varphi\right]-v_{0} v(\varphi) v^{\prime}(\varphi)|\nabla \phi|^{2} \\
-\omega_{g} d^{\prime}(\varphi)+p^{\prime}(\phi) T_{0}^{-1} \kappa \gamma_{0}(\nabla \cdot \mathbf{u}), \\
\kappa \nabla_{i}\left(\nabla \cdot \mathbf{u}-\gamma_{0} p(\varphi)\right)+\mu \nabla_{j} \nabla_{j} u_{i}=0 .
\end{gathered}
$$

The equation for the orientation field $(\phi)$ remains unchanged. Fourier transform permits rewriting equation (24) as

$$
\kappa k_{i} k_{j} \hat{u}_{j}-i \gamma_{v} k_{i} \hat{p}+\mu k_{j} k_{j} \hat{u}_{i}=0 .
$$

Then we sum $\left(\sum_{i}\right)$ both the parts of this equation after prior multiplying by $k_{i}$. As a result, find the following expression for the Fourier components of elastic displacement:

$$
-i k_{j} \hat{u}_{j}=\frac{\gamma_{v}}{\kappa} \frac{\sum_{i}\left(k_{i}^{2} \hat{p} / \mu k_{l} k_{l}\right)}{\sum_{i}\left(k_{i}^{2} / \mu k_{l} k_{l}\right)+1} \text {. }
$$

This solution makes possible the exclusion of the displacement field from the phase field equation (23).

Consider now the isotropic 1D definition of the problem (23) and (24) under the conditions: $\varphi=0, c=c_{0}$. Suppose also that the orientation field of the whole volume is uniform $\left(\phi=\phi_{0}\right)$ and constant, and the interface orientation is $\theta=\theta_{0}$. In this case the phase field $\varphi=\varphi(z)$ describes the 1D two-phase region where the melt (with $\varphi \approx 0$ ) corresponds to $z \rightarrow \infty$, and the crystal (with $\varphi \approx 1$ ) agrees to $z \rightarrow-\infty$. It is anticipated that the interphase region where $\varphi$ is varied between 0 and 1 is located near $z=0$.

For the conditions of the imposed two-axis deformation $e_{x x}=e_{y y}=e_{0}$ we have from (22) for the stress tensor components $\left(\sigma_{x x}, \sigma_{y y}\right)$ :

$$
\sigma_{x x}=\sigma_{y y}=\frac{18 \kappa \mu}{3 \kappa+4 \mu}\left(e_{0}-\gamma_{0}\right)
$$

Accordingly, the following expression can be written for the elastic energy potential:

$$
f_{e l}=\frac{18 \kappa \mu}{3 \kappa+4 \mu}\left(e_{0}-\gamma_{0}\right)^{2} .
$$

Hence, the following modification of MPF can be obtained from (17)-(20):

$$
\varepsilon_{0}^{2}\left(\theta_{0}\right) \frac{d^{2} \varphi}{d x^{2}}=\frac{d f}{d \varphi}
$$

where 


$$
f=\omega T d(\varphi)+\left[L\left(1-T / T_{m}\right)+\frac{18 \kappa \mu}{3 \kappa+4 \mu}\left(e_{0}-\gamma_{0}\right)^{2}\right] p(\varphi) \text {. }
$$

After that multiplying both the sides of (25) by $d \varphi / d x$ and performing one integration, derive

$$
\frac{1}{2} \varepsilon_{0}^{2}\left(\frac{d \varphi}{d x}\right)^{2}-f(\varphi, T)=f(0, T)=f(1, T) .
$$

From (26) follows the expression for the equilibrium temperature

$$
\frac{L\left(T-T_{0}\right)}{T_{0}}=-\frac{2 \mu^{S}\left(3 \lambda^{S}+2 \mu^{L}\right)}{\lambda^{S}+2 \mu^{L}}\left(e_{0}-\gamma_{0}\right)^{2} .
$$

Equation (27) characterizes the influence of elastic fields on the equilibrium temperature. The solution of (26) with the boundary condition $f(0, T)=0$ has the form:

$$
x-x_{0}=\sqrt{\varepsilon_{0}} \int_{1 / 2}^{\varphi} \frac{d \varphi^{\prime}}{\sqrt{2 f\left(\varphi^{\prime}, T\right)}} .
$$

\section{Macro-scale model}

The macro-scale model of SLM describes the dynamics of variation of the macroscopic fields of the temperature, velocities, pressure, as well as the evolution of the melt free surface. The knowledge of these fields is necessary in solving the microproblem to define the phase fields during crystallization. Fig. 1 shows the schematic diagram of the SLM. In the formulation of the macro-scale model the following assumptions have been made: the Gaussian and "top-hat" distributions of laser beamintensity are considered; laser radiation, when absorbed in the powder layer on the substrate, generates a microscopic region of melt having a certain depth and width; it also induces the emergence of surface forces causing the melt motion owing to the thermocapillary effect at the cost of the temperature gradient; consideration is given to the radiation intensities $\left(J_{0}\right)$ whereby evaporation of the powder particles is practically absent. Since metals are intensively evaporated at the temperatures $T>T_{v}$, where $T_{v}$ is the temperature of metal evaporation (at atmospheric pressure), evaporation-free regimes are obtainable over a wide range of temperatures $T_{m}<T<T_{v}$. Newtonian liquids are considered; all physical properties of the liquid except surface tension do not depend on the

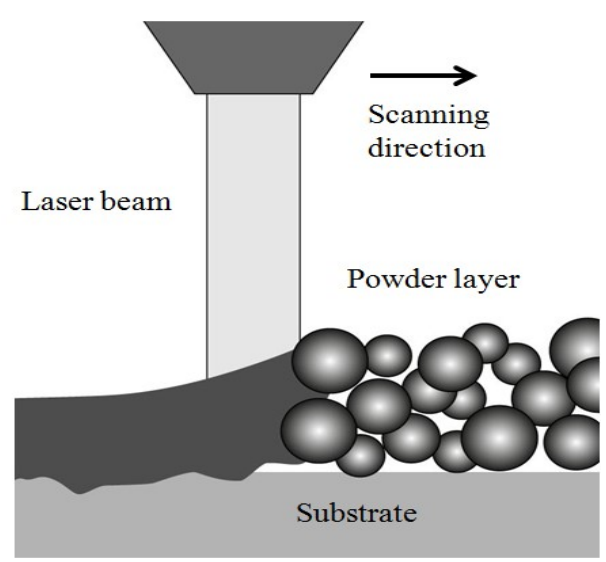

Fig. 1. SLM of a powder bed temperature.

The macro-scale model involves the coupled equations of: continuity

$$
\frac{\partial \rho}{\partial t}+\nabla \cdot(\rho \mathbf{v})=0
$$

Navier-Stokes

$$
\frac{\partial \rho \mathbf{v}}{\partial t}+\nabla(\rho \mathbf{v v})-\operatorname{div}(\mu \nabla \mathbf{v})=-\nabla P-\frac{\mu}{K} \mathbf{v}
$$

energy transfer

$$
\frac{\partial(\rho h)}{\partial t}+\nabla \cdot(\rho \mathbf{v} h)=\nabla \cdot(k \nabla T)-\nabla \cdot\left(\rho f_{S}\left(h_{L}-h_{S}\right) \mathbf{v}\right)+Q_{\text {las }},
$$

where $\mathbf{v}=(u, v, w)$ is the liquid velocity vector; $P$ the hydrodynamic pressure; $\mu=\mu_{L} \rho / \rho_{L}$ the viscosity; $h, k$ and $\rho$ are the medium enthalpy, heat conduction and density, respectively; $Q_{\text {las }}=\beta_{\text {las }} J$ is the intensity of the volume heat source associated with laser action at different depths of the powder layer ( $J$ and $\beta_{\text {las }}$ are the density of laser radiation energy flow and the radiation absorption coefficient in the local volume of the powder layer, respectively). $f_{S}(\mathbf{r}, t)=1-f_{L}(\mathbf{r}, t)$, $f_{L}(\mathbf{r}, t)=M_{L} / M_{0}$ is the mass fraction of the liquid phase formed at the point $\mathbf{r}=(x, y, z)$ by the moment of time $t\left(M_{0}\right.$ and $M_{L}$ are the total masses of metal and liquid phase, respectively). $f_{L}=0$ for the solid phase, $f_{L}=1$ for the totally transformed phase and for the two-phase region $0<f_{L}<1$. In the two-phase region (mushy zone) under study, the mass fraction of the liquid phase is defined by the formula $f_{L}=\left(1+\rho_{S} g_{S} / \rho_{L} g_{L}\right)^{-1}$, here $g_{L}, \rho_{L}$ and $g_{S}, \rho_{S}$ are the volume fractions and densities of the liquid and solid phases.

The second term in the right side of (29) allows for the variation of angular momentums at the cost of liquid filtration through the porous medium (Darcy law), where $K$ is the medium permeability, $K=K_{0}\left(f_{L}^{3}+10^{-10}\right)\left(1-f_{L}\right)^{-2}$, $\left(K_{0}\right.$ is the empiric constant defined by the interface morphology). $K \rightarrow 0$ corresponds to the purely solid phase $\left(f_{L}=0\right)$. In this case the 
Darcy term becomes large, and the velocity of liquid decays to zero. $K \rightarrow \infty$ corresponds to the purely liquid phase $f_{L}=1$, when the Darcy term disappears.

The second term in the left side of (30) deals with convective heat transfer. The first term in the right side of (30) characterizes the transfer of heat at the cost of heat conduction; the second term describes the energy flow connected to relative movement of the $L$ and $S$ phases. Taking account of the heat flow released by laser radiation is introduced in the model as a volume source $Q_{\text {las }}$ proportional to the volume absorption coefficient. The absorption accompanying laser radiation penetration into the powder layer is described by the law similar to Bouguer law for the optically uniform media: $J=J_{0} \exp \left(-\beta_{\text {Las }} z\right)$, where $J_{0}$ is the density of the energy flow on the layer surface.

Considering that the enthalpies of the solid and liquid phases are $h_{S}=c_{S} T$ and $h_{L}=c_{L} T+\left(c_{S}-c_{1}\right) T_{S}+L_{m}$, respectively, and the mass fraction of the liquid phase is linearly dependent on the temperature $f_{L}=\left(T-T_{S}\right)\left(T_{L}-T_{S}\right)^{-1}, T_{S} \leq T \leq T_{L}\left(T_{S}\right.$ and $T_{L}$ are the solidus and liquidus temperatures, respectively), the energy equation is written as

$$
\frac{\partial\left(\rho c_{L} T\right)}{\partial t}+\mathbf{v} \nabla \cdot\left(\rho c_{L} T\right)-\nabla \cdot(k \nabla T)=-\frac{\partial\left(\rho f_{L} L\right)}{\partial t}+\frac{\partial\left(\rho f_{S} \Delta c_{p} T\right)}{\partial t},
$$

where the source term in the right side represents a variation in the enthalpy related to PT.

In the two-phase zone the density, velocity vector, enthalpy and heat conduction are found by the values of the volume and mass fractions [15]

$$
\rho_{m}=\rho_{S} g_{S}+\rho_{L} g_{L}, \quad \mathbf{v}=\mathbf{v}_{S} f_{S}+\mathbf{v}_{L} f_{L}, k_{m}=g_{S} k_{S}+g_{L} k_{L}, h_{m}=h_{S} f_{S}+h_{L} f_{L} .
$$

The boundary conditions on the free surface (liquid-gas interface) allow for convective $\left(q_{H}\right)$ and radiation loss $\left(q_{T}\right)$ :

$$
-k \nabla T=q_{H}+q_{T}, \quad q_{H}=-h_{c}\left(T-T_{0}\right), \quad q_{T}=-\sigma_{E} \sigma_{S B}\left(T^{4}-T_{0}^{4}\right),
$$

where $h_{c}$ is the coefficient of convective heat transfer; $T_{0}$ is the air temperature; $\sigma_{E}$ is the surface emission coefficient; $\sigma_{S B}$ is Stefan-Boltzmann constant.

At the interface of the liquid and solid phases $\mathbf{v}=0$, which agrees with the conditions of non-leakage (the velocity component, normal to the surface, is zero) and adherence (the tangential component of velocity is zero). On the free surface of the liquid the capillary $\left(\mathbf{F}_{c}\right)$ and Marangoni $\left(\mathbf{F}_{M}\right)$ forces act, which are due to the surface tension gradient associated with the temperature field inhomogeneity along the interface (thermo-capillary forces):

$$
\mathbf{F}_{S / L}=\mathbf{F}_{c}+\mathbf{F}_{M}=\gamma \kappa \mathbf{n}+\nabla_{\tau} \gamma
$$

where $\kappa=-(\nabla \cdot \mathbf{n})$ is the free surface curvature; $\mathbf{n}$ is the vector of free surface normal, $\nabla_{\tau}$ is the surface gradient operator. For most condensed media: $\gamma(T)=\gamma_{0}\left(T_{m}\right)-\gamma_{T}\left(T-T_{m}\right), \gamma_{T}=\left|\partial \gamma_{0} / \partial T_{m}\right|$. Accordingly, for the total surface force we have

$$
\mathbf{F}_{S / L}=\gamma \kappa \mathbf{n}+\gamma_{T}(\nabla T-\mathbf{n}(\mathbf{n} \cdot \nabla T)) .
$$

In the course of SLM the shape of the free surface (the interphase boundary gas-liquid/solid) is changed because of convective flows of the liquid on the surface. To define the evolution of the free surface, make use of the transport equation for the liquid volume fraction $(\alpha)$ in the micro-region (VOF model) that has the form [16]

$$
\frac{\partial \alpha}{\partial t}+\mathbf{v} \cdot \nabla \alpha=0
$$

If $\alpha=1$, the microregion is completely filled with metal, if $\alpha=0$, it is filled with gas. If $0<\alpha<1$, the micro-region contains a free surface. It is evident that $\alpha+\beta=1$, where $\beta$ is the volume fraction of the gas phase.

The physical properties of the medium in the transition zone (gas-liquid/solid) is found by the VOF function

$$
\begin{aligned}
& \rho=\rho_{g}+\alpha\left(\rho_{m}-\rho_{g}\right), \quad c_{p}=c_{p g}+\alpha\left(c_{p m}-c_{p g}\right), \quad \lambda=\lambda_{g}+\alpha\left(\lambda_{m}-\lambda_{g}\right), \\
& h=h_{g}+\alpha\left(h_{m}-h_{g}\right), \quad \mu=\mu_{g}+\alpha\left(\mu_{m}-\mu_{g}\right),
\end{aligned}
$$

the values with $m$ index refer to the metal and the values with $g$ index belong to the gas.

The surface forces $\left(\mathbf{F}_{S / L}\right)$, acting per unit of free surface area can be transformed to the volume forces by Dirac delta function $\delta(\alpha)$, i.e.

$$
\mathbf{F}_{S / L}^{v o l}=\mathbf{F}_{S / L} \delta(\alpha)=\gamma \kappa \nabla \alpha+\gamma_{T}(\nabla T-\mathbf{n}(\mathbf{n} \cdot \nabla T))|\nabla \alpha| .
$$

Accordingly, for the volume sources in the energy equation we have:

$$
q^{v o l}=\left(q_{H}+q_{L}\right) \delta(\alpha)+Q_{\text {las }} \text {. }
$$

The normal to the free surface is found by the gradient of VOF function: $\mathbf{n}=\nabla \alpha /|\nabla \alpha|$, and its location is defined with the help of VOF function itself.

The set of equations (28)-(31) represents a macroscopic thermodynamic model of SLM. In combination with the appropriate boundary conditions it permits defining the temperature distribution, the velocity fields of thermo-capillary flows and the profile of the melt free surface depending on the regimes (beam power, scanning velocity) of the SLM process.

For the purpose of numerical calculation using the outlined model, the program software has been developed. Its realization involved the $\mathrm{C}++$ class library of numerical modeling OpenFOAM2.4. The finite volume method was applied on the unstructured hexahedral mesh [17]. 
Computer Optics and Nanophotonics / F.Kh. Mirzade, A.V. Dubrov

The initial powder layer was applied by specifying the nonuniform original structure of the field of metal phase volume fraction $(\alpha)$. The powder particles were given as the distributed spherical regions. The PISO algorithm was used to solve the continuity and Navier-Stokes equations for the liquid dynamics. The transport equation for the volume fraction is calculated by the MULES method [18]. The energy equation is solved by the "enthalpy - porosity" method using the implicit scheme [19].

The $3 \mathrm{D}$ distribution of the volume fraction of the metal phase in the operating region has been obtained. Fig. 2 presents the examples of calculating the evolution of the metal phase field structure under the scanning action of laser radiation of $200 \mathrm{~W}$ power on the pre-poured layer of the particles of Inconel 718 powder (the particle size $40 \mu \mathrm{m}$, the scanning velocity $1.7 \mathrm{~m} / \mathrm{s}$ ).

Fig. 2 displays the specific effects accompanying the SLM process - melting of the metal particles, wetting of the solid metal with the melt, coalescence of the liquid droplets. The calculated distributions also demonstrate the development of widespread defects in SLM technology, e.g. residual porosity inside the solidified metal in the form of gas bubbles, incomplete penetration and bonding of the substrate metal and the particles. The obtained results suggest that the capillary effects make a decisive contribution to the dynamics of the liquid phase and, correspondingly, to the final profile and structure of the deposed layer.

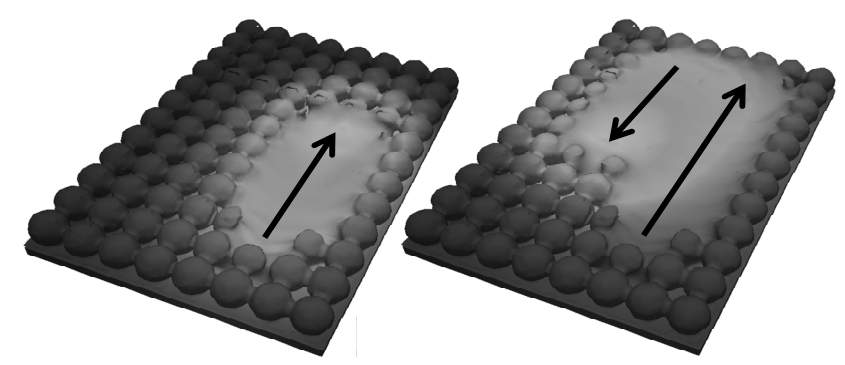

Fig 2. The distribution of the metal phase during scanning two consecutive tracks.

\section{Conclusion}

The mathematical statement of the problem of crystallization and evolution of solid phase microstructure in the course of SLM of a powder compact has been formulated and substantiated by the use of two-scale approximation. The microstructure formation is described by the equation for the two-component ordering parameter (degree of order, orientation field), conjugate with the equations of energy transfer and impurity diffusion, as well as by the elastic stresses accompanying PT. In formulating the elasticity equation the constitutive relations were used which relate the elastic stresses to the fields of strains, temperature, concentration, as well as to the ordering parameter. The model under study was constructed on basis of the unified entropy functional and the law of its increment (entropy production positivity) that is also valid for non-isothermal conditions, which agrees with the principles of thermodynamics of irreversible processes. The particular cases of the derived evolution equations have been discussed.

The model of microstructure is adjoint with the macroscopic thermodynamic model of SLM. At the macrolevel, taking account of the heat transfer processes, thermocapillary convection and free surface evolution receives primary attention. The macromodel can find application in forecasting thermal flows and melt velocity fields depending on the technological parameters (beam power, scanning velocity) of the SLM process. Modeling of the free surface evolution involved the application of the VOF function. The data obtained from the macrolevel (e.g., heat removal rate) can be used as the input parameters in formulating the boundary conditions for solving the microproblem as well. The macroproblem has been numerically realized, and the test calculation of the metal phase distribution has been conducted.

The developed model can provide the basis for predictive investigation of the formation of microstructure and stress-strain states which is requisite for the control and optimization of the additive SLM technologies of synthesis of polycrystalline materials.

\section{Acknowledgments}

The work has been performed with the support of the Russian Federation for Basic Research (grant no: 16-29-11743 ofi-m).

\section{References}

[1] Gladush GG, Smurov I. Physics of Laser Materials Processing: Theory and Experiment. Berlin: Springer-Verlag, 2011 ; 534 p.

[2] Xiao B, Zhang Yu. Laser sintering of metal powders on top of sintered layers under multiple-line laser scanning. J Phys. D: Appl. Phys. 2007; 40: 67256732 .

[3] Gusarov AV, Yadroitsev I, Bertrand Ph, Smurov I. Heat transfer modelling and stability analysis of selective laser melting. Appl. Surf. Sci. 2007; 254: 975-983.

[4] Modern laser and information technologies. Edited by acad V. Ya. Panchenko and prof F.V. Lebedev. M.: Intercontact Nauka, 2014 ; 959 p.

[5] Markl M., Korner C. Multi-Scale modeling of powder-bed-based additive manufacturing. Annual Review of Materials Research 2016 ; 46 : 1-34.

[6] Mirzade FKh. Phase field approach to solidification including stress effects at laser sintering of metal powders. J. Applied Spectroscopy 2017; 84(8) (accepted). 
[7] Dubrov AV, Dubrov VD, Mirzade FKh, Panchenko VYa. Heat transfer and thermocapillary convection in laser additive manufacturing process by injection of metal powders. Poverchnost 2017 (accepted).

[8] Wang J, Yang M, Zhang Yu. A multiscale nonequilibrium model for melting of metal powder bed subjected to constant heat flux. Int. J. Heat and Mass Transfer 2015; 80: 309-318.

[9] Wang S-L, Sekerka RF, Wheeler AA, et al. Thermodynamically-consistent phase-field models for solidification. Physica D 1993; 69: 189-200.

[10] Warren JA, Boettinger WJ. Prediction of dendritic growth and microsegregation patterns in a binary alloy using the phase-field method. Acta Metall. Mater. 1995; 43: 689-703.

[11] Bi Z, Sekerka RF. Phase-field model of solidification of a binary alloy. Physica A 1998; 261: 95-106.

[12] Penrose O, Fife PC. Thermodynamically consistent models of phase-field type for the kinetics of phase transitions. Physica D 1990; 43: 44-62.

[13] Karma A, Rappel W-J. Quantitative phase-field modeling of dendritic growth in two and three dimensions. Phys. Rev. E 1998; 57: 4323-49.

[14] Kobayashi R, Warren JA, Carter WC. Vector-valued phase field for crystallization and grain boundary formation. Phys. D 1998; 119: 415-423.

[15] BennonWD, Incropera FP. A continuum model for momentum, heat and species transport in binary solid-liquid phase change systems. I. Model formulation. Int. J. Heat Mass Transfer 1987; 30: 2161-2169.

[16] Osher S, Sethian JA. Fronts propagation with curvature-dependent speed: algoritms based on Hamilton-Jacobi formulations. J. Comput. Phys. 1988; 79: $12-49$.

[17] Weller HG, Tabor G, Jasak H, Fureby C. A tensorial approach to computa-tional continuum mechanics using object orientated techniques. Comput. Phys. 1998; 12: 620-631.

[18] Marquez Damian S. An Extended Mixture Model for the Simultaneous Treatment of Short and Long Scale Interfaces: Doctor Thesis. Santa Fe: Universidad Nacional Del Litoral, 2013; 231 p.

[19] Voller VR, Prakash C. A fixed grid numerical modelling methodology for convection-diffusion mushy region phase-change problems. Int. J. of Heat and Mass Transfer 1987; 30(8): 1709-1719. 